HIP-2010-14/TH

IPM/P-2010/033

\title{
Light-Like Noncommutativity, Light-Front Quantization and New Light on UV/IR Mixing
}

\author{
M. M. Sheikh-Jabbari ${ }^{1}$ and A. Tureanu ${ }^{2}$ \\ ${ }^{1}$ School of Physics, Institute for research in fundamental sciences (IPM), \\ P.O.Box 19395-5531, Tehran, Iran* and \\ ${ }^{2}$ Department of Physics, University of Helsinki and Helsinki Institute of Physics, \\ P.O.Box 64, FIN-00014 Helsinki, Finland
}

\begin{abstract}
We revisit the problem of quantizing field theories on noncommutative Moyal spacetime with light-like noncommutativity. To tackle the issues arising from noncommuting and hence nonlocal time, we argue that for this case light-front quantization procedure should be employed. In this appropriate quantization scheme we perform the non-planar loop analysis for the light-like noncommutative field theories. One of the important and peculiar features of light-front quantization is that the UV cutoff of the light-cone Hamiltonian manifests itself as an IR cutoff for the light-cone momentum, $p^{+}$. Due to this feature, the naive results of covariant quantization for the light-like case allude to the absence of the UV/IR mixing in the light-front quantization. However, by a careful analysis of non-planar loop integrals we show that this is not the case and the UV/IR mixing persists. In addition, we argue in favour of the perturbative unitarity of light-like noncommutative field theories in the light-front quantization scheme.

PACS numbers: 11.30.Cp, 03.30.+p, 11.10.Nx
\end{abstract}

*Electronic address: jabbari@theory.ipm.ac.ir

$\dagger$ Electronic address: anca.tureanu@helsinki.fi 


\section{INTRODUCTION}

The notion of the usual (Lorentzian) space-time is expected to break down due to quantum gravity effects. A simple way to model such quantum effects in space-time, which is appearing in certain brane and string theory settings [1], is a space-time with noncommuting coordinate operators

$$
\left[x^{\mu}, x^{\nu}\right]=i \theta^{\mu \nu}
$$

where $\theta^{\mu \nu}$ is in general a function of $x^{\mu}$. In usual treatments, which are again based on the picture coming from string theory settings, $\theta^{\mu \nu}$ is a given matrix and is related to the vacuum expectation value of a background Kalb-Ramond two form field [2]. As a result, the Lorentz symmetry on noncommutative (NC) space-time is broken to one of its subgroups.

Lorentz invariance has been at the heart of the formulation of quantum field theories (QFTs), with their fundamental properties such as causality, unitarity, spin-statistics and CPT theorems, and the very notion of labeling the states (and the fields) by representations of the Poincaré group. The lack of Lorentz invariance could then jeopardize the pillars of QFT. Noncommutative QFT with constant $\theta^{\mu \nu}$, though Lorentz-noninvariant, is symmetric under the subgroup of Lorentz group which leaves invariant the matrix $\theta^{\mu \nu}$, be it $O(1,1) \times S O(2)$ for space-like noncommutativity $\left(\theta_{0 i}=0\right), S O(1,1) \times S O(2)$ for time-like

noncommutativity $\left(\theta_{0 i} \neq 0, \theta_{\mu \nu} \theta^{\mu \nu}<0\right)[3]$ or $T(2)$ for the light-like case $\left(\theta_{\mu \nu} \theta^{\mu \nu}=0\right)$. However, using the quantum-group concept of Abelian twist [4] and relying on the translational invariance of $\mathrm{NC}$ space-time with constant $\theta^{\mu \nu}$, it was shown that $\mathrm{NC}$ QFT has a more subtle symmetry, under the twisted Poincaré algebra [5-7]. This symmetry ensures that the particle representations of NC QFT are identical with the representations of the Poincaré algebra, thus preserving the labeling of the quantum states according to their mass and spin.

Equipped with the notion of twist, one can study QFTs on NC space-times (1) with constant $\theta^{\mu \nu}$ and still rely on the CPT and spin-statistics theorems, which have been shown to hold [8-10]. The action for NC QFTs is obtained from the commutative counterpart by replacing the usual product of functions (fields) with the nonlocal Moyal $\star$-product:

$$
(\phi \star \psi)(x)=\phi(x) e^{\frac{i}{2} \theta^{\mu \nu} \overleftarrow{\partial_{\mu}} \overrightarrow{\partial_{\nu}}} \psi(x)
$$

The infinite nonlocality in the NC directions induced by the $\star$-product can be an imped- 
iment for quantization. The nonlocality in time is decisive: space-like NC field theories can be quantized, while theories with NC time can not be quantized either in interaction picture, or in Heisenberg picture or in path integral formulation [11]. This appears as a by-product of the causality and unitarity analysis: it has been shown that time-like NC field theories, upon naive covariant quantization, do not respect (perturbative) unitarity [12], nor causality [13], while space-like and light-like NC QFTs have been argued to be unitary $[3,12,14]$.

Recently, light-like NC QFTs have been shown to be relevant in another context, the Very Special Relativity [15]. In [15], Cohen and Glashow put forward the idea of a master theory at high energies, which includes the effects of quantum gravity, preserving translational invariance but breaking the Lorentz symmetry (and recovering it at lower energies), under the name of Very Special Relativity. They argued that one may relate the violation of the timereversal, $T$, and charge-conjugation times parity, $C P$, symmetries of particle physics models with a possible violation of Lorentz invariance. This can happen noting that there are subalgebras of Lorentz algebra which, upon the action of $T$ and $C P$ on the generators, will close on the whole Lorentz algebra (and not a subalgebra of it). One can show that there are only four such possible subalgebras, the Very Special Relativity algebras, T(2), E(2), HOM(2) and $S I M(2)$ [15]. All of them are rank two and have only one-dimensional representations.

In [16] we showed that NC and VSR invariant space-times are related to each other: $T(2)$ algebra and translational invariance is the symmetry of a NC space-time with light-like noncommutativity $\left(\theta_{\mu \nu} \theta^{\mu \nu}=0\right)$ and constant $\theta^{\mu \nu}$ (light-like Moyal plane); the corresponding NC QFTs are then formulations of T(2) VSR quantum field theories. Actually, the other three VSR subalgebras of Lorentz can also be realized as symmetries of a light-like NC spacetime, with certain $x$-dependent noncommutativity parameter $\theta^{\mu \nu}(x)$, but with the violation of (a part of) translational invariance, and thus physically not attractive.

In this new context, it is natural to investigate in more depth the light-like noncommutative cases, especially with regard to the possibility of applying a consistent quantization approach.

It is interesting that, while being nonlocal in time, the light-like case has turned out not to have a unitarity problem. The latter would ultimately be related to the lack of a well-defined time-ordering needed for computation of QFT correlation functions. This could presumably be traced to a special feature in the light-like case, which other NC field theories with nonlocal time do not share: in the light-cone frame, $\theta^{\mu \nu}$ has only $\theta^{-i}$ components, 
where $i=1,2$ denotes the space directions transverse to the light-cone, and hence the lightcone time coordinate $x^{+}$is commuting with the other coordinates. In this sense, light-like noncommutative field theories in the light-front coordinates (infinite-momentum frame, [1719]) are similar to the space-like noncommutative cases. In other words, the quantization of light-like noncommutative cases can be performed in the light-cone frame without a timeordering ambiguity. For a review on light-front quantization, see [20].

Having invoked the canonical quantization procedure for NCQFTs, one can perform loop calculations. The effects of the NC phase resulting from the $\star$-product $(2)$ in momentum space does not show up in the tree level two point functions, but the interaction terms are modified and hence, in general the NC effect appears in the so-called non-planar loop Feynman diagrams [21]. This momentum-dependent phase factor improves the UV divergent behavior of the non-planar loop diagrams, while giving rise to the $U V / I R$ mixing phenomenon: sending the UV cutoff to infinity leads to the appearance of undealt with IR divergences. The UV/IR mixing seems to be a ubiquitous feature of all NCQFTs [21, 22] and persists beyond one loop [23].

In a nutshell, while the loop momentum of the planar diagrams is regularized by a cutoff $\Lambda$, according to the standard result of [21], in the non-planar loop diagrams we have a corresponding effective cutoff $\Lambda_{\text {eff }}$ given by

$$
\Lambda_{e f f}^{2}=\frac{1}{1 / \Lambda^{2}+p \circ p}
$$

where $p$ is the external momentum and $p \circ p=\left(p^{\mu} \theta_{\mu \nu}\right)^{2}$. The UV/IR mixing then arises from the fact that upon taking the limit $\Lambda \rightarrow \infty$, the effective cutoff becomes $\Lambda_{\text {eff }}^{2}=\frac{1}{p \circ p}$, which obviously goes to infinity in the limit $p \rightarrow 0$ (IR limit). It should, however, be noted that the expression (3) has been obtained performing the loop integrals in the Wick rotated Euclidean frame. This procedure, while being correct for space-like noncommutative cases, as we have argued above and due to the subtleties with time-ordering, will not be reliable for the lightlike noncommutative cases. Here, we show by explicit computations using the light-cone time-ordering and light-front quantization, which is the appropriate quantization scheme, that for the light-like cases the dependence of $\Lambda_{\text {eff }}$ on external momenta is more complicated than the one given in (3) and in particular the dependence on the external momenta is not only a function of $p \circ p$. In fact, if (3) were true for the light-like noncommutative case we would have had good reason to believe that we do not have UV/IR mixing for the light-like 
case. As we shall show in sections II and III, $p \circ p$ depends only on the light-cone momentum $p^{+}$, which is regularized in the discrete light-cone quantization (DLCQ) so that $p \circ p \sim \Lambda^{-2}$; this could have led to the absence of UV/IR mixing.

Through a careful analysis of non-planar diagrams in the light-like case in the DLCQ, which is the appropriate quantization scheme, we show that the non-planar Feynmann diagrams do not remain IR finite when the UV cutoff of the theory is removed and hence the UV/IR mixing problem persists. Using our one loop results we argue for the unitarity of the theory at this level.

\section{DISCRETE LIGHT-CONE QUANTIZATION AND NC QFT}

To study the noncommutative non-planar loop diagrams we consider the $\lambda \phi_{\star}^{4}$ theory with the action

$$
S=\int d^{4} x\left[\frac{1}{2} \partial_{\mu} \phi \partial^{\mu} \phi-\frac{1}{2} m^{2} \phi^{2}-\frac{\lambda}{4 !} \phi \star \phi \star \phi \star \phi\right] .
$$

In the light-cone frame,

$$
d s^{2}=2 d x^{+} d x^{-}-d x_{i}^{2}, \quad \partial_{\mu} \phi \partial^{\mu} \phi=2 \partial_{+} \phi \partial_{-} \phi-\partial_{i} \phi \partial_{i} \phi, \quad i=1,2,
$$

where $x^{ \pm}=\frac{1}{\sqrt{2}}\left(x^{0} \pm x^{3}\right), x^{+}$is taken to be the light-cone time and $x^{i}$ denote transverse directions. In the light-like NC case, in this frame the only non-zero component of $\theta^{\mu \nu}$ is $\theta^{-i}$ $\left(\right.$ or $\left.\theta_{+i}\right)$,

$$
\left[x^{-}, x^{i}\right]=i \theta^{-i}
$$

In particular, we note that the $\theta^{+-}$and $\theta^{+i}$ (hence $\theta_{-+}$and $\theta_{-i}$ ) components are zero. Consequently, the light-cone time coordinate commutes with all the other directions, $\left[x^{+}, x^{-}\right]=\left[x^{+}, x^{i}\right]=0$. Note also that, due to the freedom to choose the $x_{i}$ axis in the transverse directions, there is only one physical observable related to $\theta^{-i}$, which can be taken to be $\theta^{2} \equiv \sum_{i=1,2} \theta^{-i} \theta^{-i}$.

In the space-like noncommutative case, the light-cone frame can always be taken such that the noncommutativity is only in the transverse directions, while in the time-like case the noncommutativity of light-cone time cannot be avoided. Thus, for space-like and light-like noncommutative field theories there is a well-defined time-ordering while for time-like we have time-ordering ambiguity. 
As in the perturbative treatment of any QFT, we solve the equation of motion for the quadratic part of the action, whose solutions are

$$
\phi(x)=\int \frac{d p^{+} d p_{\perp}^{2}}{\sqrt{2(2 \pi)^{3} p^{+}}}\left(\phi(p) e^{i\left(p^{+} x^{-}+p^{-} x^{+}-p_{i} x_{i}\right)}+\text { h.c. }\right)
$$

where $\vec{p}_{\perp}=\left(p^{1}, p^{2}\right)$ is the momentum transverse to the light-cone and

$$
p^{-}=\frac{p_{\perp}^{2}+m^{2}}{2 p^{+}}, \quad p_{\perp}^{2}=p_{1}^{2}+p_{2}^{2}, \quad p^{+} \geq 0 .
$$

$p^{-}$should be viewed as the (eigenvalues for) the light-cone Hamiltonian of the free theory and the non-negative $p^{+}$is the light-cone momentum, conjugated to $x^{-}$. Taking the momentum conjugated to $\phi$ to be $\frac{\partial \mathcal{L}}{\partial\left(\partial_{+} \phi\right)}=\partial_{-} \phi$, we apply the canonical quantization procedure by imposing equal light-cone time commutation relations between the field and its conjugate momentum. The commutator of the creation and annihilation operators for the scalar field is then obtained as

$$
\left[\phi\left(k^{+}, k_{i}\right), \phi^{\dagger}\left(p^{+}, p_{i}\right)\right]=\delta^{2}\left(k_{i}-p_{i}\right) \delta\left(k^{+}-p^{+}\right)
$$

It was established long ago [24] that light-front quantization of ordinary field theories leads to exactly the same physical results as in any Lorentz frame. This is a non-trivial result, if we recall that in the light-cone frame one cannot use the standard Wick rotation to perform momentum integrals. The Feynman rules for light-front QFTs are slightly different than the usual Lorentz covariant ones. These rules for general field theories and gauge field theories in particular, have been worked out and analyzed extensively (see [20] and references therein).

In the light-front quantization there is a subtlety which should be dealt with. As can be seen from (8), in the light-cone quantization there appear to be extra unphysical "spurious" poles at $p^{+}=0$, even at the tree level. To remove these unphysical poles, one may employ the "discrete light-cone quantization" (DLCQ) [20, 24]: by compactifying the $x^{-}$direction on a circle with radius $R_{-}$, the light-cone momentum $p^{+}$takes quantized values $n / R_{-}, n=$ $0,1,2, \cdots$. One may then safely remove the now isolated $p^{+}=0$ point and finally in the end of all analysis remove $R_{-}$by taking it to infinity. It is notable that $R_{-}$which is an IR regulator for $p^{+}$appears as a UV cutoff for the light-cone Hamiltonian (energy) $p^{-}$. This is an essential feature of the DLCQ, as will be seen later. 


\section{ONE LOOP ANALYSIS IN LIGHT-FRONT QUANTIZATION}

In this section we turn on interactions given by [23]

$$
\begin{aligned}
S_{\text {int }}=\frac{\lambda}{3 \cdot 4 !} \int & \prod_{n=1}^{4}\left(\frac{d^{4} k_{n}}{(2 \pi)^{4}} \phi\left(k_{n}\right)\right)(2 \pi)^{4} \delta^{4}\left(\sum_{i=1}^{4} k_{i}\right) \times \\
& \times\left[\cos \frac{k_{1} \theta k_{2}}{2} \cos \frac{k_{3} \theta k_{4}}{2}+\cos \frac{k_{1} \theta k_{3}}{2} \cos \frac{k_{2} \theta k_{4}}{2}+\cos \frac{k_{1} \theta k_{4}}{2} \cos \frac{k_{2} \theta k_{3}}{2}\right],
\end{aligned}
$$

where $k \theta p=\theta^{-i}\left(p^{+} k_{i}+k^{+} p_{i}\right)$. Consider the one loop correction to the two-point function. Using standard light-front QFT methods one can see that the loop integrals separate into two parts [21]: planar parts which do not involve the $\mathrm{NC} \cos (k \theta p / 2)$ factors and the nonplanar parts which do involve $\theta$-dependent phases. The planar diagrams have the same form as in ordinary field theory and can be regularized by a UV cutoff $\Lambda$.

In what follows, in section III A we discuss the planar and non-planar one loop correction to two point function for space-like noncommutative case in the light-front quantization and show that the result is the same as in the covariant quantization in which Wick rotation has also been used. Then, in section III B we address the same question for the light-like noncommutative case for which, as we have argued, the only consistent quantization scheme is the light-front quantization.

\section{A. Space-like NC case in light-front quantization}

Let us start with the planar two point function, for which the momentum integral ${ }^{1}$ is [23]:

$$
I_{P}=\frac{2 \lambda}{3} \cdot \frac{1}{2(2 \pi)^{3}} \int^{\Lambda} d^{2} q \int_{\epsilon}^{\Lambda} \frac{d q^{+}}{2 q^{+}},
$$

where $\Lambda$ is the UV cutoff and

$$
\epsilon=\frac{q_{i}^{2}+m^{2}}{\Lambda}, \quad i=1,2
$$

Note that even for off-shell particles running in the loops, eq. (8) is still valid and the integral is not taken over $q^{-}$. Consequently, the UV cutoff on $q^{-}$leads to an IR cutoff on

\footnotetext{
${ }^{1}$ Since we are using DLCQ prescription, instead of the integral over $q^{+}$, we should have a discrete sum. Hence, in our analysis, the $q^{+}$integral is in fact an upper bound approximation to the loop expressions.
} 
$q^{+}$. This integral can be readily performed to obtain

$$
\begin{aligned}
I_{P} & =\frac{\lambda}{3} \cdot \frac{1}{2(2 \pi)^{3}} \int^{\Lambda} d^{2} q \ln \frac{q_{i}^{2}+m^{2}}{\Lambda^{2}} \\
& =\frac{\lambda}{48 \pi^{2}}\left(\Lambda^{2}-m^{2} \ln \frac{\Lambda^{2}}{m^{2}}\right)+\text { finite terms. }
\end{aligned}
$$

For the nonplanar two-point function, taking the noncommutativity parameter in the transverse space only, $\theta^{i j} \neq 0(i, j=1,2)$, the integral becomes:

$$
I_{N P}^{S L}=\frac{\lambda}{2 \cdot 3} \cdot \frac{1}{2(2 \pi)^{3}} \int^{\Lambda} d^{2} q\left(e^{i \tilde{p}_{i} q_{i}}+e^{-i \tilde{p}_{i} q_{i}}\right) \int_{\epsilon}^{\Lambda} \frac{d q^{+}}{2 q^{+}},
$$

where $\tilde{p}_{i}=\theta \epsilon_{i j} p_{j}$. We can perform the integral by replacing the IR and UV cutoffs by exponentials, so that the $q^{+}$integral becomes

$$
I^{+}=\int_{0}^{\infty} \frac{d q^{+}}{q^{+}} e^{-\frac{q^{+}}{\Lambda}-\frac{\epsilon}{q^{+}}}=2 K_{0}\left(\frac{2}{\Lambda} \sqrt{q^{2}+m^{2}}\right) .
$$

One then performs the angular part of the two-dimensional $q_{i}$ integral to obtain a Bessel function, yielding

$$
I_{N P}^{S L}=\frac{\lambda}{24 \pi^{2}} \int_{0}^{\Lambda} d q q J_{0}(|\tilde{p}| q) K_{0}\left(\frac{2}{\Lambda} \sqrt{q^{2}+m^{2}}\right),
$$

where $|\tilde{p}|^{2}=\theta^{2}\left|p_{\perp}\right|^{2}$. Noting that for $q \gg \Lambda$ the $K_{0}$ function already involves an exponential damping and that $J_{0}$ for large $q$ grows like $q^{-1 / 2}$, one may relax the UV cutoff and use Formula 6.596-7 of [25] to arrive at

$$
\begin{aligned}
I_{N P}^{S L} & =\frac{\lambda}{24 \pi^{2}} \int_{0}^{\infty} d q q J_{0}(|\tilde{p}| q) K_{0}\left(\frac{2}{\Lambda} \sqrt{q^{2}+m^{2}}\right) \\
& =\frac{\lambda}{48 \pi^{2}} m^{2} \cdot \frac{\Lambda_{e f f}}{m} K_{1}\left(\frac{2 m}{\Lambda_{e f f}}\right),
\end{aligned}
$$

where $\Lambda_{\text {eff }}^{-2}=\Lambda^{-2}+\frac{|\tilde{p}|^{2}}{4}$. This expression is exactly the same as what one would get using the standard Wick rotation and doing Euclidean integrals [21].

\section{B. Light-like NC case in light-front quantization}

As in the usual light-front quantization [24] and as we have explicitly showed above, the planar diagrams are the same as the ones in the covariant quantization. We hence focus on the non-planar diagrams. The non-planar diagrams of the light-like case have to be 
computed anew in the light-front quantization. Using the standard light-front Feynman rules, the corresponding non-planar two-point function is

$$
\mathcal{I}_{N P}^{L L}=I_{N P}^{L L}+\left(I_{N P}^{L L}\right)_{c . c .}
$$

where

$$
\begin{gathered}
I_{N P}^{L L}=\frac{\lambda}{12 \cdot(2 \pi)^{3}} \int^{\Lambda} d^{2} q e^{i \alpha_{i} q_{i}} \int_{\epsilon}^{\Lambda} \frac{d q^{+}}{2 q^{+}} e^{i \beta q^{+}}, \\
\alpha_{i}=\theta^{-i} p^{+}, \quad \beta=\theta^{-i} p_{i},
\end{gathered}
$$

and $\left(I_{N P}^{L L}\right)_{c . c .}=I_{N P}^{L L}\left(-\alpha_{i},-\beta\right)$.

One of the peculiar features of light-front field theory is that to remove the pole at $p^{+}=0$, usually DLCQ procedure [20] is invoked, i.e. we remove all the physical states with $p^{+}$below $1 / R_{-}$. Recalling (8), this implies a lower bound on the UV cutoff of the light-cone energy $m^{2} /\left(1 / R_{-}\right)$. In performing the loop integrals we impose the UV cutoff $\Lambda$ on all momenta (a universal cutoff) and hence the IR tree-level cutoff $R_{-}$and the UV loop-level cutoffs are not independent and they should be chosen such that

$$
\Lambda \sim m^{2} R_{-}, \quad \text { or } \quad \frac{\Lambda}{R_{-}} \sim m^{2}
$$

In other words, the IR cutoff on the light-cone momentum running in the loops is indeed the one in (12).

We perform the $q^{+}$integral by replacing the IR and UV cutoffs by exponentials, using the Formula 3.471-9 of [25]:

$$
\begin{aligned}
\int_{\epsilon}^{\Lambda} \frac{d q^{+}}{q^{+}} e^{i \beta q^{+}} & =\int_{0}^{\infty} \frac{d q^{+}}{q^{+}} e^{-\frac{q^{+}}{\Lambda}} e^{-\frac{\epsilon}{q^{+}}} e^{i \beta q^{+}} \\
& =2 K_{0}\left(\frac{2 \sqrt{1-i \beta \Lambda}}{\Lambda} \sqrt{q^{2}+m^{2}}\right) .
\end{aligned}
$$

Computing the angular integral of the transverse $2 D$ part we obtain

$$
\begin{aligned}
I_{N P}^{L L} & =\frac{\lambda}{96 \pi^{2}} \int_{0}^{\infty} q K_{0}\left(2 \sqrt{1-i \beta \Lambda} \sqrt{\frac{q^{2}+m^{2}}{\Lambda^{2}}}\right) J_{0}(|\alpha| q) d q \\
& =\frac{\lambda}{2 \cdot 96 \pi^{2}} m^{2} \frac{\Lambda_{e f f}}{m} K_{1}\left(\frac{2 m}{\Lambda_{e f f}}\right)
\end{aligned}
$$

where

$$
\Lambda_{\text {eff }}^{-2}=\Lambda^{-2}+\frac{|\alpha|^{2}}{4}-\frac{i \beta}{\Lambda}, \quad|\alpha|^{2}=\theta^{2}\left(p^{+}\right)^{2}
$$


A proof of the above result which is based on the Formulae 6.596-7 and 8.535 of [25] is given in the Appendix A. The complete non-planar integral (18) is then

$$
\mathcal{I}_{N P}^{L L}=I_{N P}^{L L}\left(\Lambda_{e f f}\right)+I_{N P}^{L L}\left(\Lambda_{e f f}^{*}\right)
$$

After exponentiating the IR and UV cutoffs on $q^{+}$, one can perform the $q^{i}$ integral first and then the $q^{+}$integral, and as expected we get the same result as above.

When $|z|=\left|\frac{m}{\Lambda_{e f f}}\right|$ is very small, one may approximate $\frac{2}{z} K_{1}(2 z) \simeq z^{-2}+\ln z^{2}$ and arrive at

$$
I_{N P}^{L L}=\frac{\lambda}{2 \cdot 96 \pi^{2}}\left(\Lambda_{e f f}^{2}-m^{2} \ln \frac{\Lambda_{e f f}^{2}}{m^{2}}\right)
$$

and, by using (24), we obtain $\mathcal{I}_{N P}^{L L}$ in terms of the real part and the absolute value of $\Lambda_{\text {eff }}^{2}$ :

$$
\mathcal{I}_{N P}^{L L}=\frac{\lambda}{96 \pi^{2}}\left[\left(\Lambda_{e f f}^{2}\right)_{R e}-m^{2} \ln \frac{\left|\Lambda_{e f f}^{2}\right|}{m^{2}}\right],
$$

where

$$
\begin{aligned}
\left(\Lambda_{\text {eff }}^{2}\right)_{R e} & =\left(1+\frac{\Lambda^{2}|\alpha|^{2}}{4}\right)\left[\frac{1}{\Lambda^{2}}\left(1+\frac{\Lambda^{2}|\alpha|^{2}}{4}\right)^{2}+\beta^{2}\right]^{-1}, \\
\left|\Lambda_{e f f}^{2}\right| & =\Lambda\left[\frac{1}{\Lambda^{2}}\left(1+\frac{\Lambda^{2}|\alpha|^{2}}{4}\right)^{2}+\beta^{2}\right]^{-1 / 2} .
\end{aligned}
$$

Due to the appearance of the $\beta$ terms, the non-planar light-like loop integral (25), unlike the non-planar space-like noncommutative result [21], is not simply a function of only $p \circ p=$ $|\alpha|^{2}=\theta^{-i} \theta_{+i}\left(p^{+}\right)^{2} \equiv \theta^{2} p^{2}$.

\section{$U V / I R$ discussion}

To study the UV/IR connection one should analyze $\left|\Lambda_{\text {eff }}^{2}\right|$ and $\left(\Lambda_{\text {eff }}^{2}\right)_{R e}$ in the $\Lambda \rightarrow \infty$ limit, while the external momenta are held fixed [21]. In our case, however, as we discussed, we have the peculiar feature that the minimum value of the external light-cone momentum is indeed linked with the UV cutoff $\Lambda$. In particular, (21) implies that

$$
\Lambda|\alpha| \gtrsim m^{2} \theta
$$


which must be taken into account when taking $\Lambda \rightarrow \infty$, while $\beta=\theta^{-i} p_{i}$ is held fixed. Therefore, after sending the UV cutoff $\Lambda$ to infinity one obtains:

$$
\begin{aligned}
\left(\Lambda_{e f f}^{2}\right)_{R e} & \simeq\left(1+\frac{m^{4} \theta^{2}}{4}\right) \frac{1}{\left(\theta^{-i} p_{i}\right)^{2}}, \\
\left|\Lambda_{e f f}^{2}\right| & \simeq \frac{\Lambda}{\theta^{-i} p_{i}}
\end{aligned}
$$

Taking now the $p_{i} \rightarrow 0$ limit in (28), we see that the expressions show IR divergences.

If the limits are taken in the opposite order, i.e. first the $p \rightarrow 0$ limit, we obtain, as in the case of space-space noncommutativity, $\Lambda_{e f f}=\Lambda[21]$. Again, the limits do not commute, and the UV/IR mixing persists. To confirm the validity of our present result, in the Appendix $\mathrm{B}$ we have performed the integral (19) using other regularization methods.

\section{Discussion on the unitarity of the light-like NCQFT}

Based on string theory results, it has been argued [14] that light-like NC field theories are unitary, in the sense of having a unitary S-matrix. Time-like NC cases are, however, non-unitary as they do not satisfy the Cutkosky rules (and the optical theorem) [12]. A simple way to address the unitarity issue is to consider the one loop effective action focusing on the two point function result, which in the standard case (with $\Lambda_{\text {eff }}$ given by (3)) is

$$
S_{1 P I}=\int d^{4} p \frac{1}{2}\left[p^{2}-M^{2}+\frac{\lambda}{96 \pi^{2}} \frac{1}{p \circ p}\right] \phi(p) \phi(-p)
$$

where $M$ and $\phi(p)$ are respectively renormalized mass and field. The unitarity of the theory at this one-loop level could be physically traced to the existence (or absence) of ghosts: if $p \circ p$ is positive (negative) the kinetic term will remain positive (or can become negative). We next recall that $p \circ p$ is positive for space-like or light-like noncommutative cases while is negative for time-like noncommutative case.

As we have shown, in the light-like case one should invoke light-front quantization and the expression for the one-loop non-planar diagram changes. Nonetheless, from (28) it is evident that the first term in (25) is positive definite and hence one expects the light-like noncommutative field theories in light-front quantization to be unitary field theories. 


\section{DISCUSSION}

We have argued that the quantization of field theories with light-like noncommutativity is consistent only in the light-front quantization approach. The argument is based on the fact that the light-cone time $x^{+}$is commutative $\left(\left[x^{+}, x^{-}\right]=\left[x^{+}, x^{i}\right]=0, \quad i=1,2\right)$, therefore it is local and the canonical quantization procedure can be directly applied. The spacespace noncommutative field theories can also be quantized using the light-front formalism, while for time-space noncommutativity the light-cone time is nonlocal, just like the physical time, and the light-front method is expected to be no more consistent than the covariant quantization.

We have performed the one-loop analysis and showed that in the case of space-space noncommutativity, the light-front quantization and the covariant quantization give, as expected, exactly the same results. It is expected that this equivalence hold to all orders.

Prompted by the hint given by the covariant quantization analysis and in particular that the effective cutoff of the theory for non-planar loops is given by (3), one could have hoped that the UV/IR mixing were absent in the light-like noncommutative case in the discrete light-cone quantization approach. However, through a rigorous light-front study of the one-loop two point function of the $\lambda \phi_{\star}^{4}$, we have shown that this is not the case and the non-planar diagrams exhibit IR divergences. Here, to illustrate this point, we have presented a two point function analysis at one loop. It is, however, easy to see that the non-planar one-loop four point function is controlled by similar "effective cutoffs" (26). We expect this one loop result to be carried to all loops order. Moreover, it is expected that, as in the space-like noncommutative case [23], the noncommutativity parameter $\theta$ receive no loop corrections at higher order in perturbation.

We have also briefly discussed the unitarity of light-like noncommutative field theories and argued that based on our one-loop two-point function result, we expect these theories to be (perturbatively) unitary and free of ghosts. This point deserves a more thorough study.

In our discussions we have considered the four-dimensional $\lambda \phi_{*}^{4}$ theory. The results and, in particular, the persistence of UV/IR mixing are expected to be true for any light-like NC quantum field and gauge field theories. Our calculations show that light-front quantization in the light-like NC field theories leads to different expressions compared to the covariant quantization approach. This may bring some new features and results which are not seen in 
the covariant approach. Loop analysis should be revisited using the light-front quantization, with special emphasis on the NC QED loop effects and the chiral anomaly in chiral NC gauge field theories.

As shown in [16], light-like noncommutative field theories are a framework for formulating VSR-invariant field theories. It is hence interesting to study the implications of our results and analysis in this context.

\section{Acknowledgements}

We are grateful to Masud Chaichian for useful discussions and comments. We also thank José Gracia-Bondía and Peter Prešnajder for comments on the manuscript. The support of the Academy of Finland under the projects no. 121720 and 127626 is acknowledged.

\section{Appendix A: More details on the integral in Eq. (22)}

In computing the integral in eq. (20) Formula 6.596-7 of [25] has been used. This formula, however, as it stands only applies to real argument for Bessel-K and Bessel-J functions, while in eq. (22) we have complex valued arguments. Below we shall show that for our case and using another formula of [25] together with 6.596-7 one can prove eq. (22). To start with, let us recall that the integral we want to take is of the form

$$
\mathcal{I}=\int_{0}^{\infty} q K_{0}\left(|\gamma| e^{i \Phi} \sqrt{q^{2}+m^{2}}\right) J_{0}(|\alpha| q) d q
$$

where

$$
\gamma^{2} \equiv|\gamma|^{2} e^{2 i \Phi}=\frac{4}{\Lambda^{2}}(1 \pm i \beta \Lambda)
$$

From the above, we learn that $-\frac{\pi}{2} \leq 2 \Phi \leq \frac{\pi}{2}$. Next, recalling Formula 8.535 of [25],

$$
\begin{aligned}
K_{0}\left(|\gamma| e^{i \Phi} \sqrt{q^{2}+m^{2}}\right)= & \sum_{n=0}^{\infty} \frac{1}{n !} K_{n}\left(|\gamma| \sqrt{q^{2}+m^{2}}\right) \times \\
& \times\left(\frac{1-e^{2 i \Phi}}{2}|\gamma|\right)^{n}\left(q^{2}+m^{2}\right)^{n / 2}
\end{aligned}
$$

the integral takes the form

$$
\begin{aligned}
\mathcal{I} & =\sum_{n=0}^{\infty} \frac{1}{n !}\left(\frac{1-e^{2 i \Phi}}{2}|\gamma|\right)^{n} \\
& \times \int_{0}^{\infty} J_{0}(|\alpha| q) K_{-n}\left(|\gamma| \sqrt{q^{2}+m^{2}}\right)\left(q^{2}+m^{2}\right)^{n / 2} q d q
\end{aligned}
$$


where in the above we have used the fact that $K_{-n}(z)=K_{n}(z)$. We should also comment that in using Formula 8.535 of [25] we need to make sure that $\left|1-e^{i \Phi}\right|^{2}<1$. This condition is fulfilled if $\cos \Phi>\frac{1}{2}$, which includes our case where $-\frac{\pi}{4} \leq \Phi \leq \frac{\pi}{4}$.

The integral in (A4) can now be safely taken using Formula 6.596-7 of [25] to obtain

$$
\mathcal{I}=\frac{m}{\sqrt{|\alpha|^{2}+|\gamma|^{2}}} \sum_{n=0}^{\infty} \frac{1}{n !}\left(\frac{1-\lambda^{2}}{2}\right)^{n} Z^{n} K_{n+1}(Z)
$$

where

$$
\begin{aligned}
Z & =m \sqrt{|\gamma|^{2}+|\alpha|^{2}} \\
\lambda^{2} & \equiv 1-\left(1-e^{2 i \Phi}\right) \frac{|\gamma|^{2}}{|\alpha|^{2}+|\gamma|^{2}}=\frac{|\alpha|^{2}+\gamma^{2}}{|\alpha|^{2}+|\gamma|^{2}}
\end{aligned}
$$

In the above we have used the notation $\gamma^{2}=|\gamma|^{2} e^{2 i \Phi}$. Iff $|1-\lambda|^{2}<1$, then one can resum the above series using Formula 8.535 of [25] to obtain

$$
\begin{aligned}
\mathcal{I} & =\frac{m}{\sqrt{|\alpha|^{2}+\gamma^{2}}} K_{1}\left(m \sqrt{|\alpha|^{2}+\gamma^{2}}\right) \\
& =m^{2} \frac{\Lambda_{\text {eff }}}{2 m} K_{1}\left(\frac{2 m}{\Lambda_{e f f}}\right)
\end{aligned}
$$

where

$$
\Lambda_{e f f}^{-2}=\Lambda^{-2}+\frac{|\alpha|^{2}}{4}-\frac{i \beta}{\Lambda} .
$$

The only point one should check to complete the proof is whether $|1-\lambda|^{2}<1$ holds. If $\beta \Lambda=x,|\alpha| \Lambda=2 y$, then $\lambda=|\lambda| e^{i \psi}$, where

$$
|\lambda|^{2}=\frac{\sqrt{\left(y^{2}+1\right)^{2}+x^{2}}}{y^{2}+\sqrt{x^{2}+1}}, \tan 2 \psi=\frac{x}{y^{2}+1} .
$$

It is then easy to see that the condition $|\lambda|<2 \cos \psi$ holds for the case of our interest in $\mathrm{UV} / \mathrm{IR}$ discussion, namely $x$ large but $y$ finite (that is, in the $\Lambda \rightarrow \infty$ limit).

\section{Appendix B: Schwinger regularization method}

Here we use another regularization method which is based on the Schwinger parametrization of the "propagator", that is by exponentiating the propagator or the expression which gives the poles. In our case,

$$
\frac{1}{q^{+}}=\int_{0}^{\infty} d x e^{-x q^{+}}
$$


The UV and IR cutoffs on $q^{+}$, respectively $\Lambda$ and $\epsilon$, then turn to IR and UV cutoffs on $x$, namely $1 / \Lambda$ and $1 / \epsilon$. Let us now perform the $q^{+}$integral:

$$
\begin{aligned}
I^{+} & =\int_{\epsilon}^{\Lambda} \frac{d q^{+}}{q^{+}} e^{-i \beta q^{+}}=\int_{\frac{1}{\Lambda}}^{\frac{1}{\epsilon}} d x \int_{0}^{\infty} d q^{+} e^{-x q^{+}} e^{-i \beta q^{+}} \\
& =\int_{\frac{1}{\Lambda}}^{\frac{1}{\epsilon}} d x \frac{d x}{x+i \beta}=-\ln \frac{\epsilon}{\Lambda}+\ln \frac{1+i \beta \epsilon}{1+i \beta \Lambda}
\end{aligned}
$$

where $\beta$ is defined in (20) and

$$
\epsilon=\frac{q_{i}^{2}+m^{2}}{\Lambda}
$$

We can now perform the $q$-integrals. There are two such integrals:

$$
\begin{aligned}
\mathcal{I}_{1} & =\int^{\Lambda} d^{2} q e^{i \alpha_{i} q^{i}} \ln \frac{q^{2}+m^{2}}{\Lambda^{2}}=\int^{\Lambda} d^{2} q e^{i \alpha_{i} q^{i}} \int_{\epsilon}^{\Lambda} \frac{d q^{+}}{q^{+}} \\
& =2 \pi m^{2} \cdot \frac{\Lambda_{\text {eff }}}{m} K_{1}\left(\frac{2 m}{\Lambda_{e f f}}\right),
\end{aligned}
$$

where

$$
\Lambda_{e f f}^{-2}=\Lambda^{-2}+\frac{|\alpha|^{2}}{4} .
$$

This part of the integral is $\beta$-independent.

Next, let us consider the $\beta$-dependent $q$-integral:

$$
\begin{aligned}
I_{\beta} & =\int^{\Lambda} d^{2} q e^{i \alpha_{i} q^{i}} \ln \frac{1+\beta^{2} \epsilon^{2}}{1+\beta^{2} \Lambda^{2}} \\
& =\pi \int^{\Lambda} q d q J_{0}(|\alpha| q) \ln \frac{1+\beta^{2} \epsilon^{2}}{1+\beta^{2} \Lambda^{2}}
\end{aligned}
$$

which can be rewritten as

$$
\begin{aligned}
I_{\beta} & =\pi \Lambda^{2} \int_{0}^{1} y d y J_{0}(a y) \ln \frac{1+b^{2} \rho^{2}}{1+b^{2}} \\
& \simeq \pi \Lambda^{2} \int_{0}^{\infty} y d y J_{0}(a y) e^{-y^{2}} \ln \frac{1+b^{2} \rho^{2}}{1+b^{2}}
\end{aligned}
$$

where

$$
a=|\alpha| \Lambda, \quad b=\beta \Lambda, \quad \rho=y^{2}+\frac{m^{2}}{\Lambda^{2}}
$$

We may use either of (B4a) or (B4b) to continue the analysis; here we focus on (B4a). 
In order to perform the $y$-integrals of $I_{\beta}$ it turns out to be more convenient to integrate the expression before adding its complex conjugate. We use the series expansion

$$
\begin{aligned}
\ln \frac{1+i b \frac{\epsilon}{\Lambda}}{1+i b} & =\ln \left(1-\frac{i b}{1+i b}\left(1-\frac{\epsilon}{\Lambda}\right)\right) \\
& =-\sum_{n=1}^{\infty} \frac{1}{n}\left(\frac{i b}{1+i b}\right)^{n}\left(1-y^{2}-\frac{m^{2}}{\Lambda^{2}}\right)^{n}
\end{aligned}
$$

where $b=\beta \Lambda$ and $y=q / \Lambda$. Then,

$$
I_{\beta}=\hat{I}_{\beta}+\hat{I}_{\beta}^{c . c .},
$$

where

$$
\hat{I}_{\beta}=-\pi \Lambda^{2} \sum_{n=1}^{\infty} \frac{1}{n}\left(\frac{i b}{1+i b}\right)^{n}\left(\sum_{k=0}^{n}\left(\begin{array}{l}
n \\
k
\end{array}\right)\left(-\frac{m^{2}}{\Lambda^{2}}\right)^{k} A_{n-k}(a)\right)
$$

with (cf. Formula 6.683 .6 of [25])

$$
A_{n}(a)=\int_{0}^{1} d y \text { y } J_{0}(a y)\left(1-y^{2}\right)^{n}=\frac{2^{n} n !}{a^{n+1}} J_{n+1}(a) .
$$

We obtain

$$
\hat{I}_{\beta}=-\pi \Lambda^{2} \sum_{n=1}^{\infty}(n-1) !\left(\frac{2 i b}{1+i b}\right)^{n} \sum_{k=0}^{n} \frac{1}{k !}\left(-\frac{m^{2}}{2 \Lambda^{2}}\right)^{k} \frac{J_{n-k+1}(a)}{a^{n-k+1}}
$$

and hence

$$
\begin{aligned}
I_{\beta}=-\pi \Lambda^{2} \sum_{n=1}^{\infty} & (n-1) !\left(\frac{2 b}{1+b^{2}}\right)^{n}\left[(b+i)^{n}+(b-i)^{n}\right] \\
& \times \sum_{k=0}^{n} \frac{1}{k !}\left(-\frac{m^{2}}{2 \Lambda^{2}}\right)^{k} \frac{J_{n-k+1}(a)}{a^{n-k+1}}
\end{aligned}
$$

or

$$
\begin{gathered}
I_{\beta}=-2 \pi \Lambda^{2} \sum_{n=1}^{\infty}(n-1) !\left(\frac{2 b^{2}}{1+b^{2}}\right)^{n}\left[\sum_{j=0}^{\left[\frac{n}{2}\right]} \frac{(-1)^{j}}{b^{2 j}}\left(\begin{array}{c}
n \\
2 j
\end{array}\right)\right] \\
\times \sum_{k=0}^{n} \frac{1}{k !}\left(-\frac{m^{2}}{2 \Lambda^{2}}\right)^{k} \frac{J_{n-k+1}(a)}{a^{n-k+1}} .
\end{gathered}
$$

\section{UV/IR analysis}

In order to detect the UV/IR mixing in the $\beta$-sector we study the limit

$$
\Lambda \rightarrow \infty, \quad \beta=\text { fixed }
$$


In this limit, $I_{\beta}$ decomposes into $\Lambda^{2}$ terms and $\Lambda^{0}$ terms:

$$
\begin{aligned}
I_{\beta} & =-\pi \Lambda^{2} \sum_{n=1}^{\infty}(n-1) ! 2^{n+1} \frac{J_{n+1}(a)}{a^{n+1}} \\
& +\pi \sum_{n=1}^{\infty}(n-1) ! 2^{n}\left(m^{2} \frac{J_{n}(a)}{a^{n}}+\frac{n(n+1)}{\beta^{2}} \frac{J_{n+1}(a)}{a^{n+1}}\right)+\cdots
\end{aligned}
$$

The $\Lambda^{2}$ term, which should be subtracted off in e.g. MS regularization, is simply $b$ independent. Note that $J_{n}(a) / a^{n}$ remains finite at small and large $a$ and the sum over $n$ is convergent and finite. The $\Lambda^{0}$ term has $1 / \beta^{2}$. This $\beta$ dependence leads to the IR divergence in $p_{i} \rightarrow 0$ limit and hence $\mathrm{UV} / \mathrm{IR}$ mixing. This confirms the result we presented in section III B, in eqs. (25) and (26).

[1] N. Seiberg and E. Witten, "String theory and noncommutative geometry," JHEP 9909, 032 (1999), [arXiv:hep-th/9908142].

[2] F. Ardalan, H. Arfaei and M. M. Sheikh-Jabbari, "Noncommutative geometry from strings and branes," JHEP 9902, 016 (1999), [arXiv:hep-th/9810072].

[3] L. Álvarez-Gaumé, J. L. F. Barbón and R. Zwicky, "Remarks on time-space noncommutative field theories," JHEP 0105, 057 (2001), [arXiv:hep-th/0103069].

[4] V. G. Drinfel'd, "Hamiltonian structures of Lie groups, Lie bialgebras and the geometric meaning of the classical Yang-Baxter equations," Sov. Math. Dokl. 27, 68 (1983).

[5] M. Chaichian, P.P. Kulish, K. Nishijima and A. Tureanu, "On a Lorentz-invariant interpretation of noncommutative space-time and its implications on noncommutative QFT,"Phys. Lett. B 604, 98 (2004), [arXiv:hep-th/0408069].

[6] M. Chaichian, P. Prešnajder and A. Tureanu, "New concept of relativistic invariance in NC space-time: Twisted Poincare symmetry and its implications," Phys. Rev. Lett. 94, 151602 (2005), [arXiv:hep-th/0409096].

[7] M. Chaichian, K. Nishijima, T. Salminen and A. Tureanu, "Noncommutative Quantum Field Theory: A Confrontation of Symmetries," JHEP 0806, 078 (2008), [arXiv:0805.3500[hep-th]].

[8] M. M. Sheikh-Jabbari, "Discrete symmetries (C,P,T) in noncommutative field theories," Phys. Rev. Lett. 84, 5265 (2000), [arXiv:hep-th/0001167]. 
[9] M. Chaichian, K. Nishijima and A. Tureanu, "Spin-statistics and CPT theorems in noncommutative field theory," Phys. Lett. B 568, 146 (2003), [arXiv:hep-th/0209008].

[10] A. Tureanu, "Twist and spin-statistics relation in noncommutative quantum field theory," Phys. Lett. B 638, 296 (2006), [arXiv:hep-th/0603219]; "Twisted Poincare symmetry and some implications on noncommutative quantum field theory," Prog. Theor. Phys. Suppl. 171 34 (2007), [arXiv:0706.0334[hep-th]].

[11] K. Fujikawa, "Path integral for space-time noncommutative field theory," Phys. Rev. D 70, 085006 (2004), [arXiv:hep-th/0406128].

[12] J. Gomis and T. Mehen, "Space-time noncommutative field theories and unitarity," Nucl. Phys. B 591, 265 (2000), [arXiv:hep-th/0005129].

[13] N. Seiberg, L. Susskind and N. Toumbas, "Space/Time Non-Commutativity and Causality," JHEP 0006, 044 (2000), [arXiv:hep-th/0005015].

[14] O. Aharony, J. Gomis, T. Mehen, "On theories with light-like noncommutativity," JHEP 0009, 023 (2000), [arXiv:hep-th/0006236].

[15] A. G. Cohen and S. L. Glashow, "Very special relativity," Phys. Rev. Lett. 97, 021601 (2006), [arXiv:hep-ph/0601236].

[16] M. M. Sheikh-Jabbari and A. Tureanu, "Realization of Cohen-Glashow Very Special Relativity on Noncommutative Space-Time," Phys. Rev. Lett. 101, 261601 (2008), [arXiv:0806.3699 [hep-th]].

[17] S. Fubini and G. Furlan, "Renormalization effects for partially conserved currents," Physics 1, 229 (1965).

[18] S. Weinberg, "Dynamics at Infinite Momentum," Phys. Rev. 150, 1313 (1966)

[19] L. Susskind, "Model of Self-Induced Strong Interactions," Phys. Rev. 165, 1535 (1968).

[20] S. J. Brodsky, H. C. Pauli and S. S. Pinsky, "Quantum Chromodynamics and Other Field Theories on the Light Cone," Phys. Rept. 301, 299 (1998), [arXiv:hep-ph/9705477].

[21] S. Minwalla, M. Van Raamsdonk and N. Seiberg, "Noncommutative perturbative dynamics," JHEP 0002, 020 (2000), [arXiv:hep-th/9912072].

[22] A. Matusis, L. Susskind and N. Toumbas, "The UV/IR connection in the non-commutative gauge theories," JHEP 0012, 002 (2000), [arXiv:hep-th/0002075].

[23] A. Micu and M. M. Sheikh-Jabbari, "Noncommutative $\Phi^{4}$ theory at two loops," JHEP 0101, 025 (2001), [arXiv:hep-th/0008057]. 
[24] S. J. Chang, R. G. Root and T. M. Yan, "Quantum field theories in the infinite momentum frame. 1. Quantization of scalar and Dirac fields," Phys. Rev. D 7, 1133 (1973).

S. J. Chang and T. M. Yan, "Quantum field theories in the infinite momentum frame. 2. Scattering matrices of scalar and Dirac fields," Phys. Rev. D 7, 1147 (1973).

A. Casher, "Gauge Fields On The Null Plane," Phys. Rev. D 14, 452 (1976).

[25] I.S. Gradshteyn and I.M. Ryzhik, Table of Integrals, Series, and Products, 7th edition (2007), Academic Press. 